\title{
Remarks on Some Vacuum Solutions of Scalar-Tensor Cosmological Models
}

\author{
V. B. Bezerra ${ }^{1}$, C. Romero ${ }^{1}$, G. Grebot ${ }^{2}$, M. E. X. Guimarães ${ }^{2}$, and L. P. Colatto ${ }^{3,4}$ \\ 1. Departamento de Física, Universidade Federal da Paraíba, Cx. Postal 5008, 58059-970, João Pessoa, PB, Brazil \\ 2. Departamento de Matemática, Universidade de Brasília, Brasília, Brazil \\ 3. Coordenação de Partículas e Campos, Centro Brasileiro de Pesquisas Físicas, Rio de Janeiro, RJ, Brazil \\ 4. Grupo de Física Teórica José Leite Lopes, Petrópolis, RJ, Brazil
}

Received on 29 September, 2003

\begin{abstract}
We present a class of exact vacuum solutions corresponding to de Sitter and warm inflation models in the framework of scalar-tensor cosmologies. We show that in both cases the field equations reduce to planar dynamical systems with constraints. Then, we carry out a qualitative analysis of the models by examining the phase diagrams of the solutions near the equilibrium points.
\end{abstract}

\section{Introduction}

Scalar-tensor theories of gravity[1,2] represent the most natural alternatives to Einstein's theory of general relativity. The simplest and earliest scalar-tensor theory[1] considered a massless scalar field and was formulated by using as basic metric tensor the physical tensor $\tilde{g}_{\mu \nu}$, to which matter is universally coupled (Jordan-Fierz frame). Later, these theories were generalized[2] by introducing a scalar field selfinteraction and a dynamical coupling to matter.

In what concerns cosmology, the presence of a scalar field - which from now on we will call generically dilaton, has gravitational-strength couplings to matter which violate the equivalence principle. To avoid conflicts with experimental tests on the equivalence principle, it is assumed that the dilaton acquires a mass large enough that any deviations from Einstein's theory are confined on scales that are not sensitive on cosmological scales. However, a lot of work[3] has been done in the framework of low energy string theory - which is reminiscent of scalar-tensor theories of gravity - focusing in the case where the scalar field is massless. The mechanism which naturally reconciles a massless dilaton with experimental test was proposed in Ref.[4]. Indeed, a massless dilaton is shown to obey a Minimal Coupling Principle, e.g., to decouple from matter by cosmological attraction in much the same way as the generic attractor mechanism of the scalar-tensor theories of gravity[5].

On the other hand, recent observational data which contain evidence for an accelerated expansion of the universe[6] indicate that this may be induced by scalar fields which appear naturally in scalar-tensor models. Therefore, it is important to consider various possibilities of cosmological scenarios in order to study, for example, the asymptotic behaviour at late times of Friedmann-Robertson-Walker (FRW) cosmological models and to investigate if the universe evolves towards a state indistinguishable or not from the one predicted by general relativity [7].

The aim of this paper is to study vacuum solutions in the context of FRW cosmologies with flat spatial curvature $(k=0)$ for the cases of de Sitter models and warm inflation[8]. It turns out that these two cases lead to a class of field equations that can be written as planar dynamical systems plus a constraint equation.

This work is outlined as follows. In section 2, we briefly describe the scalar-tensor cosmology. In sections 3 and 4, we find the solutions of the field equations for the de Sitter and warm inflation models, respectively. Finally, in section 5 , we present some final remarks concerning our results.

\section{Scalar-Tensor Cosmology: A Brief Review}

In this section we will make a brief review of the scalartensor cosmological models and write out the field equations which we are going to deal with in the next section. We start by considering the most general scalar-tensor theories of gravity in the Jordan-Fierz frame which is given by the action

$$
\mathcal{S}=\frac{1}{16 \pi} \int d^{4} x \sqrt{-\tilde{g}}\left[\tilde{R} \Phi-\frac{\omega(\Phi)}{\Phi} \partial^{\mu} \Phi \partial_{\mu} \Phi-2 \tilde{\Lambda}(\Phi)\right]+\mathcal{S}_{m}\left[\Psi_{m}, \tilde{g}_{\mu \nu}\right]
$$


where $\tilde{g}_{\mu \nu}$ is the physical metric, $\tilde{R}$ is the curvature scalar associated to it, $\tilde{\Lambda}(\Phi)$ is a cosmological term which corresponds to the scalar field potential and $\mathcal{S}_{m}$ is the action for general matter fields which, at this point, is left arbitrary.

The physical frame of Jordan-Fierz has the disadvantage of featuring complicated evolution equations for the gravitational and scalar fields and for this reason it is more convenient to work in the Einstein (conformal) frame, in which the scalar and tensor degrees of freedom do not mix. Now, let us define two new variables: $g_{\mu \nu}$, the metric tensor in the Einstein frame, and the scalar field $\phi$. Thus, making the transformation

$$
\tilde{g}_{\mu \nu}=A^{2}(\phi) g_{\mu \nu},
$$

we decouple the two modes of propagations. This relation tells us that the metric tensor in the Einstein frame is conformally related to the physical metric tensor in Jordan-Fierz frame. Besides, by a redefinition of the following quantities

$$
\begin{aligned}
\Phi & =\frac{1}{G A^{2}(\phi)}, \\
\Lambda(\phi) & =A^{4}(\phi) \tilde{\Lambda}, \\
\alpha(\phi) & =\frac{d \ln A(\phi)}{d \phi},
\end{aligned}
$$

where $G$ is the bare gravitational constant and, by imposing the constraint,

$$
\alpha^{2}(\phi)=\frac{1}{[2 \omega(\phi)+3]},
$$

the new quantities $g_{\mu \nu}, \quad \phi$ and $A(\phi)$ are uniquely defined in terms of the original quantities $\tilde{g}_{\mu \nu}, \Phi, \omega(\Phi)$. In the Einstein frame, the action (1) turns into

$$
\mathcal{S}=\frac{1}{16 \pi G} \int d^{4} x \sqrt{-g}\left[R-2 g^{\mu \nu} \partial_{\mu} \phi \partial_{\nu} \phi-2 \Lambda(\phi)\right]+\mathcal{S}_{m}\left[\Psi_{m}, A^{2}(\phi) g_{\mu \nu}\right]
$$

In this new frame the field equations read

$$
\begin{aligned}
G_{\mu \nu}+g_{\mu \nu} \Lambda(\phi) & =8 \pi G T_{\mu \nu}+2\left(\phi_{, \mu} \phi_{, \nu}-\frac{1}{2} g_{\mu \nu} \phi^{, \sigma} \phi_{, \sigma}\right), \\
\square_{g} \phi-\frac{1}{2} \frac{d \Lambda(\phi)}{d \phi} & =-4 \pi G \alpha(\phi) T
\end{aligned}
$$

where

$$
T_{\mu \nu}=\frac{2}{\sqrt{-g}} \frac{\delta \mathcal{S}_{m}}{\delta g^{\mu \nu}}
$$

with

$$
T_{\nu ; \mu}^{\mu}=\alpha(\phi) T \partial_{\nu} \phi,
$$

which means that the energy-momentum tensor in the conformal frame is no longer conserved, differently from the Jordan-Fierz frame in which the energy-momentum tensor is conserved.

In what follows we will concentrate on the FriedmannRobertson-Walker (FRW) cosmologies with flat spatial curvature $(k=0)$ and perfect-fluid matter distributions. These models are represented by a spacetime with metric

$$
d s^{2}=-d t^{2}+R^{2}(t)\left[d r^{2}+r^{2}\left(d \theta^{2}+\sin ^{2} \theta d \varphi^{2}\right)\right],
$$

being sourced by an energy-momentum tensor corresponding to a perfect fluid given by

$$
T^{\mu \nu}=(\rho+p) u^{\mu} u^{\nu}+p g^{\mu \nu},
$$

with $u^{\mu} \equiv \frac{d x^{\mu}}{d \tau}$ denoting the 4-velocity of the fluid in the Einstein frame. We can relate quantities such as density and pressure in both frames through the equations

$$
\rho=A^{4}(\phi) \tilde{\rho} \quad \text { and } \quad p=A^{4}(\phi) \tilde{p} .
$$

For the FRW models, equations (6) become

$$
\begin{aligned}
-3 \frac{\ddot{R}}{R} & =4 \pi G(\rho+3 p)+2(\dot{\phi})^{2}-\Lambda(\phi), \\
3\left(\frac{\dot{R}}{R}\right)^{2} & =8 \pi G \rho+(\dot{\phi})^{2}+\Lambda(\phi), \\
\ddot{\phi}+3 \frac{\dot{R}}{R} \dot{\phi} & =-4 \pi G \alpha(\phi)(\rho-3 p)-\frac{1}{2} \frac{d \Lambda}{d \phi} .
\end{aligned}
$$

In this work we are going to study general solutions of the above equations for two particular vacuum inflationary models: the de Sitter model (corresponding to $\Lambda(\phi)=\Lambda_{0}=$ constant) and the warm inflation model [8] (corresponding to $\Lambda(\phi)=3 \beta H^{2}$, with $H \equiv \dot{R} / R$ ). Let us define two new variables, $\psi$ and $H$, being given by

$$
\begin{aligned}
\psi & \equiv \dot{\phi} \\
H & \equiv \frac{\dot{R}}{R}
\end{aligned}
$$

In terms of these two new variables, the equations (9) for the case of de Sitter model can be written as

$$
\begin{gathered}
\dot{H}=-H^{2}-\frac{2}{3} \psi^{2}+\frac{\Lambda_{0}}{3}, \\
\dot{\psi}=-3 H \psi \\
H^{2}=\frac{\psi^{2}}{3}+\frac{\Lambda_{0}}{3}
\end{gathered}
$$

with the last equation being a constraint equation. 
On the other hand, for the warm inflation model we have

$$
\begin{gathered}
\dot{H}=(\beta-1) H^{2}-\frac{2}{3} \psi^{2}, \\
\dot{\psi}=(2 \beta-3) H \psi-3 \beta(\beta-1) \frac{H^{3}}{\psi},
\end{gathered}
$$

with the constraint equation given by

$$
H^{2}=\frac{\psi^{2}}{3(1-\beta)} .
$$

Thus, for the cases of de Sitter and warm inflation models, the field equations (9) can be written as a planar dynamical system plus a constraint equation in the variables $H$ and $\psi$, as we can conclude from the analysis of Eqs.(10)-(12) and (13)-(14), respectively.

\section{The de Sitter Model in Scalar- Tensor Cosmologies}

Let us now find analytical solutions of Eqs.(10)-(12). From Eq.(10) and the constraint Eq.(12) we get

$$
\dot{H}=\Lambda_{0}-3 H^{2},
$$

which can be integrated giving

$$
\begin{gathered}
H_{1}=\frac{\sqrt{\frac{\Lambda_{0}}{3}} \tanh \left(\sqrt{3 \Lambda_{0}}\left(t-t_{0}\right)\right)+H_{0}}{1+\sqrt{\frac{3}{\Lambda_{0}}} H_{0} \tanh \left(\sqrt{3 \Lambda_{0}}\left(t-t_{0}\right)\right)}, \\
H_{2}=\frac{\sqrt{\frac{-\Lambda_{0}}{3}} \tan \left(\sqrt{-3 \Lambda_{0}}\left(t-t_{0}\right)\right)+H_{0}}{1-\sqrt{\frac{-3}{\Lambda_{0}}} H_{0} \tan \left(\sqrt{-3 \Lambda_{0}}\left(t-t_{0}\right)\right)}, \\
H_{3}=\frac{H_{0}}{1+3 H_{0}\left(t-t_{0}\right)},
\end{gathered}
$$

for $\Lambda>0, \Lambda<0, \Lambda=0$, respectively, with $H_{0}$ being an integration constant. From Eqs.(10)-(12) and (17)-(19) we obtain the solutions $\psi(t)$ for each value of $\Lambda_{0}$. Thus, we have the following solutions $\left(H_{i}(t), \psi_{i}^{ \pm}= \pm \sqrt{3 H_{i}^{2}-\Lambda_{0}}\right)$, for $i=1,2$, and $\left(H_{3}(t), \psi_{3}= \pm \sqrt{3} H_{3}\right)$.

Let us note that the constraint equation (12) is compatible with (10) and (11). The same remark applies to the equations (13)-(15). We conclude that the constraint equations (12) and (15) are nothing but particular curves of the set of integral curves of the vector fields defined by the right-hand side of Eqs.(10), (11), (13) and (14). It can be directly verified that the first integral of the dynamical system formed by (10) and (11) is given by

$$
H^{2}=a \psi^{2 / 3}+\frac{1}{3}\left(\psi^{2}+\Lambda_{0}\right)
$$

with $a=0$ corresponding to the constraint equation (12).
Now, if a dynamical system has critical points (equilibrium points), it is often useful to investigate the behaviour of the solutions near these points. For $\Lambda>0$, Eqs.(10)-(12) admit four critical points in the phase plane $H \psi$. These points are located at $\left.A\left(\sqrt{\frac{\Lambda_{0}}{3}}\right), 0\right) ; B\left(-\sqrt{\frac{\Lambda_{0}}{3}}, 0\right) ; C\left(0, \sqrt{\frac{\Lambda_{0}}{2}}\right)$; $D\left(0,-\sqrt{\frac{\Lambda_{0}}{2}}, 0\right)$. These four points themselves represent solutions of the dynamical system formed by Eqs.(10) and (11), however only $A$ and $B$ satisfy the constraint equation given by (12). Incidentally, note that the solutions represented by $A$ and $B$ are obtained from Eq.(17) by assigning the values $H_{0}= \pm \sqrt{\frac{\Lambda_{0}}{3}}$. Moreover, $A$ and $B$ correspond(in the Einstein frame) to de Sitter cosmological models whose scale factors are given, respectively, by

$$
\begin{gathered}
R(t)=R_{0} \exp \left(\sqrt{\frac{\Lambda_{0}}{3}} t\right), \\
R(t)=R_{0} \exp \left(-\sqrt{\frac{\Lambda_{0}}{3}} t\right),
\end{gathered}
$$

where $R_{0}$ is a constant. For $\Lambda<0$ the system has no critical points, while for $\Lambda=0$ there is only one critical point at the origin $\mathrm{O}(0,0)$ of the phase plane, corresponding, modulo a rescaling of the coordinates, to the Minkowski spacetime. In all the above configurations represented by the critical points the scalar field $\phi$ is constant, since $\psi=0$.

We now will draw the phase diagrams corresponding to the solutions given by Eqs.(17)-(19) plus those represented by the critical points. The curves appearing in these diagrams represent parametric solutions $(H(t), \psi(t))$ evolving in time, in the Einstein frame. Of particular interest are the constant solutions corresponding to the equilibrium points since all the solutions considered are attracted or repelled by them. The position of the equilibrium points, when they exist, depends on the values assigned to $\Lambda_{0}$.

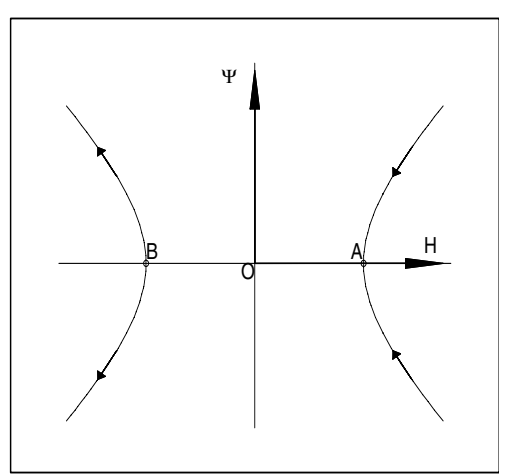

Figure 1. Phase diagram for $\Lambda_{0}>0$. The critical points $A$ and $B$ represent de Sitter universes.

The first diagram (see Fig. 1) refers to the case $\Lambda_{0}>0$. In this case we have six solutions represented by the two critical points $A$ and $B$, the two curves lying in the upper part of the hyperbolae and the two curves lying in the lower part. As we have seen before, the points $A$ and $B$ describe expanding and collapsing universes in the Einstein frame (see Eqs.(21) and (22)). At this point, it is worth noting that since $\phi=$ constant in all solutions represented 
by the points $A, B$ and $O$, we see from (2) that the metric tensor $\tilde{g}_{\mu \nu}$ is obtained from $g_{\mu \nu}$ by simply rescaling the coordinates. Thus, we have essentially the same geometry in both Einstein and Jordan-Fierz frames. It is also easy to see that, when $H_{0}>\sqrt{\frac{3}{\Lambda_{0}}}$ the point $A$ acts as an attractor for the two solutions $\left(H_{1}(t), \psi_{1}^{+}(t)\right)$ and $\left(H_{1}(t), \psi_{1}^{-}(t)\right)$ when $t \rightarrow \infty$. By reasons of continuity the same qualitative behaviour is carried over into the Jordan-Fierz frame. Quite analogously, when $H_{0}<-\sqrt{\frac{3}{\Lambda_{0}}}$, then both $\left(H_{1}(t), \psi_{1}^{+}(t)\right)$ and $\left(H_{1}(t), \psi_{1}^{-}(t)\right)$ move away from $B$ as time goes by. For $\Lambda=0$ (Fig.2) we have three solutions, which describe an expanding universe, a collapsing universe and Minkowski spacetime (represented by the equilibrium point at the origin). When $\Lambda<0$ (Fig.3) we have no equilibrium points and in the Einstein frame the solutions appear as bouncing universes possessing an expansion stage followed by a collapsing era.

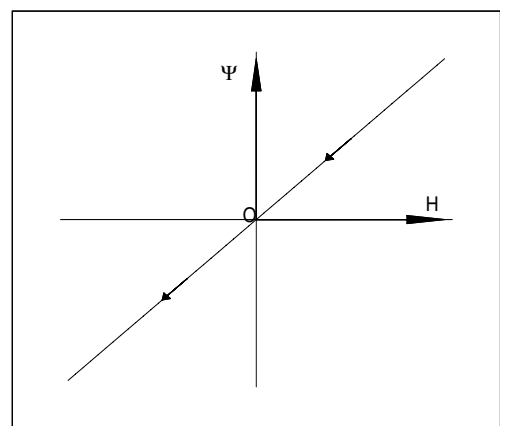

Figure 2. Phase diagram for $\Lambda_{0}=0$. Here the critical points $A$ and $B$ representing de Sitter universe merge into the origin (Minkowski spacetime).

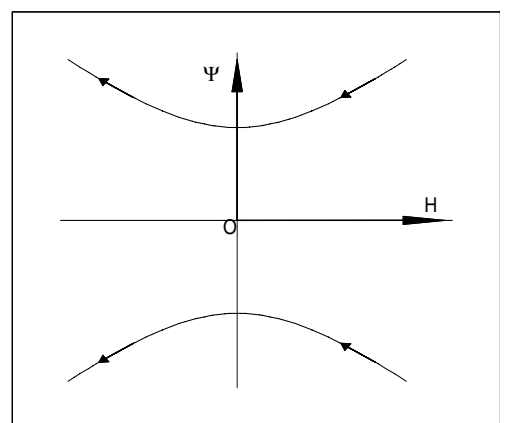

Figure 3. Phase diagram for $\Lambda_{0}<0$. The critical points disappear.

\section{The Warm Inflation Model in Scalar-Tensor Cosmologies}

Let us now consider the case of warm inflation, which corresponds to the system of equations (13)-(15). Here, the solutions for $H(t)$ and $\psi(t)$ are easily obtained if we substitute Eq.(15) into (13). This leads to

$$
\dot{H}=3(\beta-1) H^{2}
$$

the solution of which is given by

$$
H(t)=\frac{H_{0}}{1+3 H_{0}(1-\beta)\left(t-t_{0}\right)},
$$

where $H_{0}$ is an integration constant. From the constraint equation (15) we have

$$
\psi(t)= \pm \sqrt{3(1-\beta)} H(t) .
$$

Clearly the constraint equation (15) also implies that $\beta<1$. ( The case $\beta=1$ leads to $\psi=0$ and $H=H_{0}$, which describes a de Sitter universe).

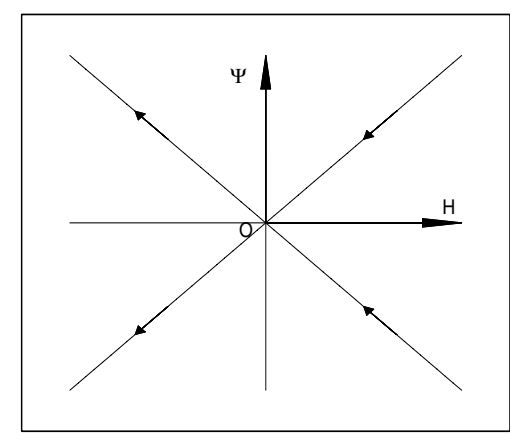

Figure 4. Phase diagram for the warm inflation model.

The phase diagram corresponding to the solutions (24) and (25) is shown in Fig. 4. These solutions represent expanding and collapsing universes, for $H>0$ and $H<0$, respectively, and Minkowski spacetime $(H=\psi=0)$. The expanding universe starts with a big-bang at $t^{*}=t_{0}+$ $\frac{1}{3 H_{0}(\beta-1)}$ and approach Minkowski spacetime as $t \rightarrow \infty$, gradually slowing their expansion rate. On the other hand, the collapsing models start at $t=-\infty$ as Minkowski spacetime and collapse at $t=t^{*}$. As we have noted in the previous section, the same qualitative analysis of the solutions in the vicinity of the origin may again be carried over into the Jordan-Fierz frame.

\section{Final remarks}

In this paper we have presented a class of exact solutions corresponding to vacuum solutions of de Sitter and warm inflation models in the context of scalar-tensor cosmology. These two particular models provide a class of field equations that can be written as planar dynamical systems plus a constraint equation.

In the case of the de Sitter model the dynamical system phase diagrams show that if $\Lambda_{0}>0$ and $\Lambda=0$ there exist solutions corresponding to critical points (de Sitter universes). It just so happens that in these cases the scalar field is constant. This fact allows us to carry over our qualitative analysis of the solutions near the equilibrium points from the Einstein frame to the Jordan-Fierz physical frame. The same remarks applies to the warm inflation model, where we have only one equilibrium point, which corresponds to Minkowski space-time. Finally, we would like to stress that we do not touch here the problem of providing a mechanism 
to terminate the inflationary phase of the universe, our solutions for both models being valid for all $t$.

\section{Acknowledgments}

V.B.B. and C.R. would like to thanks CNPq for partial financial support. V.B.B., M.E.X.G. and L.P.C. would like to thank CAPES in the context of the interinstitutional program PROCAD/CAPES for partial financial support. M.E.X.G. would like to thanks the kind hospitality of the Departamento de Física of the Universidade Federal da Paraíba where part of this work has been developed.

\section{References}

[1] M. Fierz, Helv. Phys. Acta 29, 128 (1956); P. Jordan, Z. Phys. 157, 112 (1959); C. Brans and R. H. Dicke, Phys. Rev. 24, 925 (1961)

[2] P. G. Bergmann, Int. J. Theor. Phys. 1, 25 (1968). R. V. Wagoner, Phys. Rev. D1, 3209 (1970); K. Nordtvedt, Astrophys. J. 161, 1059 (1970).

[3] D. Kalligas, K. Nordtvedt, and R. V. Wagoner, in Proceedings of the Seventh Marcel Grossmann Meeting on General
Relativity (World Scientific, 1996); A. Serna and J. M. Alimi, Phys. Rev. D50, 7304 (1996); R. V. Wagoner and D. Kalligas, in Gravitation and Gravitational Radiation, Les Houches 1995 (Cambridge, 1996); D. I. Santiago, D. Kalligas, and R. V. Wagoner, Phys. Rev. D56, 7627 (1997).

[4] T. Damour, A. M. Polyakov, Nucl. Phys. B423, 532 (1994).

[5] T. Damour, K. Nordtvedt, Phys. Rev. Lett. 70, 2217 (1993); Phys. Rev. D 48, 3436 (1993).

[6] A. G. Riess et al., Astron. J. 116 1009, (1998); S. Perlmutter et al., Ap. J. 517, 565 (1998).

[7] D. I. Santiago, D. Kalligas, and R. V. Wagoner, Phys. Rev. D 58, 124005 (1998).

[8] K. Freese, F. C. Adams, J. A. Frieman, and E. Mottola, Nucl. Phys. B287, 797 (1987); J. M. F. Maia and J. A. S. Lima, Phys. Rev. D60, 101301 (1999).

[9] A.D. Polyanin and V. F. Zaitsev, Handbook of Exact Solutions for Ordinary Differential Equations, CRC Press, 1995.

[10] J. S. R. Chrisholm and A. K. Common, J. Phys. A 20, 5459 (1987). 